\title{
Use of Raw Materials in the United States From 1900 Through 2010
}

Since the beginning of the 20th century, the types and quantities of raw materials used by U.S. manufacturers and consumers have changed over time. This fact sheet quantifies the amounts of those materials (other than food and fuel) that have been input into the U.S. economy annually for a period of 111 years, from 1900 through 2010. It provides a broad overview of all materials used but highlights the use and importance of raw nonfuel minerals in particular. This fact sheet supersedes U.S. Geological Survey Fact Sheet 2009-3008, which was published in April 2009 and covered the period 1900 through 2006.

These data have been compiled to help the public and policymakers understand the flow of raw materials used in the United States in physical terms. Such information can be helpful in assessing the past and potential effects of the materials on the environment, evaluating the materials' intensity of use, and examining the role that these materials play in the economy. It can also provide insight into what may happen to the materials at the end of their useful life.

The data are presented in three ways. Figure 1 lists the raw materials put into use annually in the United States from 1900 through 2010 based on the historical data available. The materials are divided into the following broad categories: agriculture, forestry, metals, minerals, and nonrenewable organics. Table 1 lists the amounts (by weight) of these raw materials put into use each year for the same time period. Figures 2 and 3 are graphical representations of the data in table 1. The graphs show how the expansions and contractions in raw materials usage have been coincident with major economic and military events of the past 11 decades. These events have included World War I, the Great Depression of the 1930s, World War II, the post-World War II expansion, the oil crisis of the 1970s, the recessions of the 1980s and early 1990s, the extended period of economic prosperity and technological growth at the end of the 20th century, and the global financial crisis that began in 2007.

For the purposes of this fact sheet, "usage" refers to the annual apparent inputs during the use phase of consumption (which is calculated as the sum of domestic production, imports, and recycling, minus exports). The usage data are for the first point of consumption only; ultimate consumption in the form of manufactured products could be significantly different. The data are also only for raw materials that were ready for use directly by the consumer or in the manufacture of products that were actually made and sold in

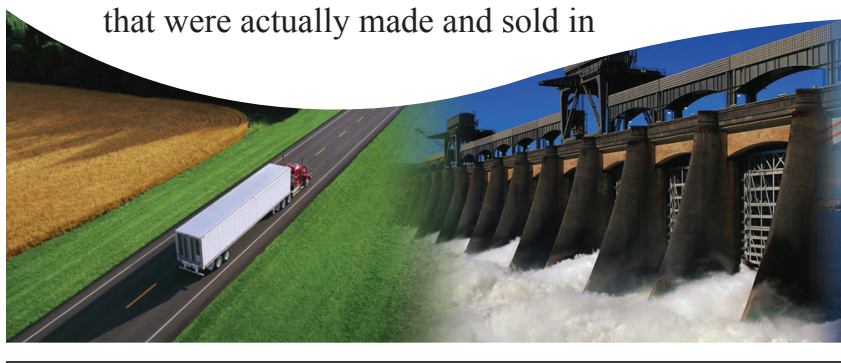

the United States, not for materials contained in finished goods that were imported into or exported out of the country. In an industrial economy such as that of the United States where the volume of goods flowing into and out of the country is large, tracking the flow of materials embedded in imported products is virtually impossible.

The following is a list of all the sources used to compile these data:

- National Oceanic and Atmospheric Administration, Fisheries of the United States (for fisheries statistics)

- Resources for the Future, Natural Resource Commodities-A Century of Statistics (for agriculture statistics)

- U.S. Bureau of Mines and U.S. Geological Survey (USGS), Mineral Resources of the United States, and Minerals Yearbook (for metal and mineral statistics)

- U.S. Census Bureau, Statistical Abstract of the United States (for agriculture statistics)

- U.S. Department of Agriculture, Agricultural Statistics (for agriculture statistics)

- U.S. Energy Information Administration, Annual Energy Review (for nonrenewable organics statistics)

- U.S. Forest Service, U.S. Timber Production, Trade, Consumption, and Price Statistics (for forestry and paper statistics)

- U.S. International Trade Commission, Synthetic Organic Chemicals (for nonrenewable organics statistics, and statistics on primary products made from petroleum and natural gas)

The USGS National Minerals Information Center provides statistics and information on the worldwide supply of, demand for, and flow of minerals and materials essential to the U.S. economy, the national security, and protection of the environment.

\section{By Grecia R. Matos}

\section{For more information, please contact:}

National Minerals Information Center U.S. Geological Survey 991 National Center Reston, VA 20192 http://minerals.usgs.gov/minerals

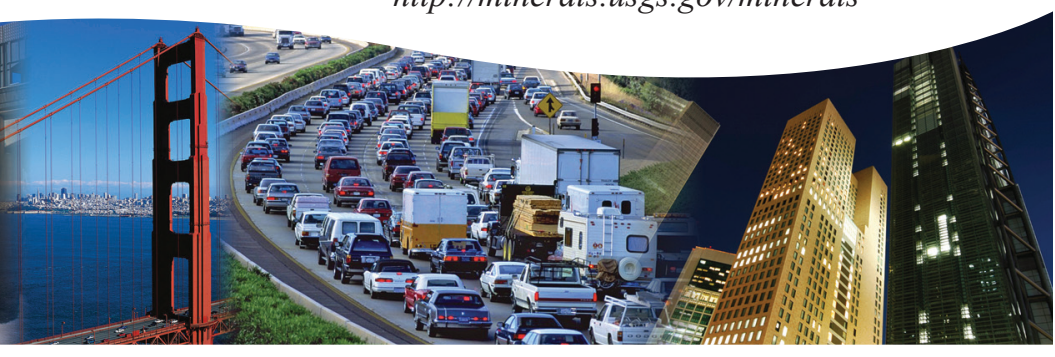




\begin{tabular}{|c|c|c|c|c|}
\hline Agriculture & Forestry & $\begin{array}{l}\text { Metals (includes } \\
\text { recycled material) }\end{array}$ & Minerals & Nonrenewable organics \\
\hline $\begin{array}{l}\text { Cotton } \\
\text { Cottonseed } \\
\text { Fishery } \\
\text { Flax seed } \\
\text { Fur } \\
\text { Leather hides } \\
\text { Mohair } \\
\text { Natural rubber } \\
\text { Raw wool } \\
\text { Silk, raw and waste } \\
\text { Tobacco }\end{array}$ & $\begin{array}{l}\text { Paper and paperboard, all grades: } \\
\text { Paper and paperboard, primary: } \\
\text { Insulating board } \\
\text { Hardboard } \\
\text { Wet machine board } \\
\text { Recycled paper, secondary, } \\
\text { 1960-2010: } \\
\text { Recovered paper at paper and } \\
\text { paperboard mills } \\
\text { Recovered paper for other uses } \\
\text { Plywood and veneer } \\
\text { Other forestry: } \\
\text { Cooperage } \\
\text { Fence posts } \\
\text { Hewn ties } \\
\text { Poles and piling } \\
\text { Other miscellaneous products } \\
\text { Wood }\end{array}$ & $\begin{array}{l}\text { Aluminum } \\
\text { Antimony } \\
\text { Arsenic } \\
\text { Beryllium } \\
\text { Bismuth } \\
\text { Cadmium } \\
\text { Cesium } \\
\text { Chromium } \\
\text { Cobalt } \\
\text { Copper } \\
\text { Gallium } \\
\text { Germanium } \\
\text { Gold } \\
\text { Indium } \\
\text { Iron and steel } \\
\text { Lead } \\
\text { Magnesium } \\
\text { Manganese } \\
\text { Mercury } \\
\text { Molybdenum } \\
\text { Nickel } \\
\text { Niobium (columbium) } \\
\text { Platinum group } \\
\text { Rare earths } \\
\text { Rhenium } \\
\text { Selenium } \\
\text { Silicon } \\
\text { Silver } \\
\text { Tantalum } \\
\text { Tellurium } \\
\text { Thallium } \\
\text { Tin } \\
\text { Titanium } \\
\text { Tungsten } \\
\text { Vanadium } \\
\text { Zinc }\end{array}$ & $\begin{array}{l}\text { Abrasives, manufactured } \\
\text { Asbestos } \\
\text { Barite } \\
\text { Boron } \\
\text { Bromine } \\
\text { Cement } \\
\text { Clays } \\
\text { Diamond, industrial } \\
\text { Diatomite } \\
\text { Feldspar } \\
\text { Fluorspar } \\
\text { Garnet, industrial } \\
\text { Gemstones } \\
\text { Graphite, natural } \\
\text { Gypsum } \\
\text { Hafnium } \\
\text { Helium } \\
\text { Iron oxide pigments } \\
\text { Kyanite and related materials } \\
\text { Lime } \\
\text { Lithium } \\
\text { Magnesium compounds } \\
\text { Mica } \\
\text { Nitrogen } \\
\text { Peat } \\
\text { Perlite } \\
\text { Phosphate } \\
\text { Potash } \\
\text { Pumice and pumicite } \\
\text { Quartz crystal } \\
\text { Salt } \\
\text { Sand and gravel, construction } \\
\text { Sand and gravel, industrial } \\
\text { Soda ash } \\
\text { Sodium sulfate } \\
\text { Stone, crushed } \\
\text { Stone, dimension } \\
\text { Strontium } \\
\text { Sulfur } \\
\text { Talc and pyrophyllite } \\
\text { Thorium } \\
\text { Titanium dioxide } \\
\text { Vermiculite } \\
\text { Wollastonite } \\
\text { Zirconium }\end{array}$ & 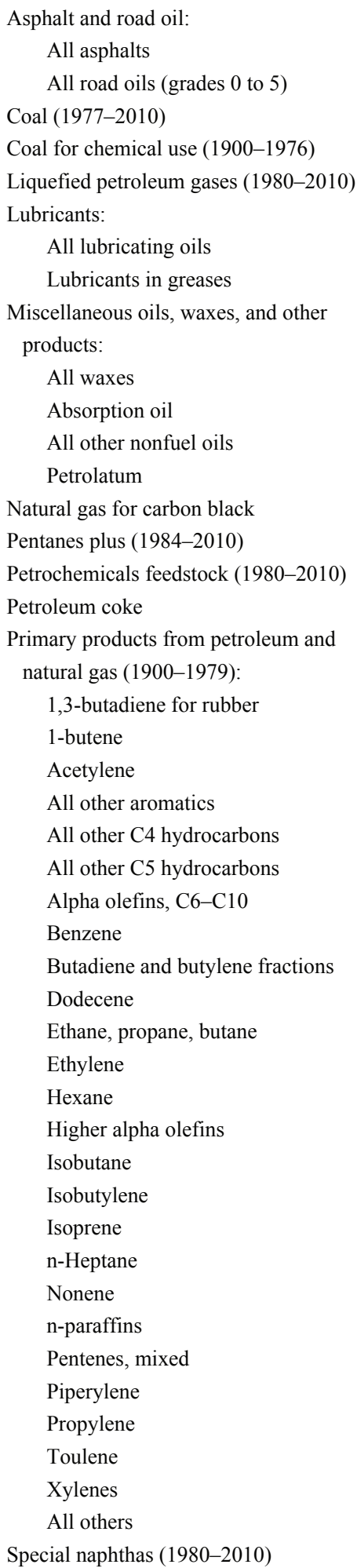 \\
\hline
\end{tabular}

Figure 1. Chart showing U.S. raw materials put into use annually from 1900 through 2010, by category. 
Table 1. U.S. raw materials put into use annually from 1900 through 2010 , by category. Materials embedded in imported goods are not included.

[In thousand metric tons. Data are rounded to three significant digits. --, negligible or no data]

\begin{tabular}{|c|c|c|c|c|c|c|c|c|c|c|}
\hline \multirow[b]{2}{*}{ Year } & \multirow[b]{2}{*}{ Agriculture } & \multicolumn{3}{|c|}{ Forestry } & \multicolumn{4}{|c|}{ Metals and minerals } & \multirow{2}{*}{$\begin{array}{c}\text { Nonrenewable } \\
\text { organics }\end{array}$} & \multirow{2}{*}{$\begin{array}{c}\text { Total of } \\
\text { materials }\end{array}$} \\
\hline & & Wood & $\begin{array}{l}\text { Paper and } \\
\text { paperboard }\end{array}$ & $\begin{array}{c}\text { Recycled } \\
\text { paper }\end{array}$ & $\begin{array}{l}\text { Primary } \\
\text { metals }\end{array}$ & $\begin{array}{c}\text { Recycled } \\
\text { metals }\end{array}$ & $\begin{array}{l}\text { Industrial } \\
\text { minerals }\end{array}$ & $\begin{array}{c}\text { Construction } \\
\text { materials }\end{array}$ & & \\
\hline 1900 & 3,040 & 60,300 & 2,640 & -- & 10,300 & -- & 10,800 & 55,300 & 1,590 & 144,000 \\
\hline 1901 & 3,460 & 62,800 & 2,670 & -- & 13,100 & -- & 11,300 & 61,000 & 1,670 & 156,000 \\
\hline 1902 & 3,660 & 65,600 & 2,690 & -- & 14,800 & -- & 13,300 & 70,000 & 1,760 & 172,000 \\
\hline 1903 & 3,300 & 67,100 & 2,720 & -- & 14,100 & -- & 14,000 & 67,000 & 1,880 & 170,000 \\
\hline 1904 & 3,560 & 69,000 & 2,750 & -- & 12,900 & -- & 20,100 & 70,100 & 1,900 & 180,000 \\
\hline 1905 & 3,680 & 70,600 & 2,720 & -- & 17,800 & -- & 23,000 & 81,200 & 1,960 & 201,000 \\
\hline 1906 & 3,740 & 75,800 & 2,690 & -- & 20,800 & 12 & 27,400 & 95,200 & 2,040 & 228,000 \\
\hline 1907 & 3,310 & 80,800 & 2,670 & -- & 21,100 & 44 & 28,700 & 90,000 & 2,130 & 229,000 \\
\hline 1908 & 3,570 & 74,200 & 2,640 & -- & 12,900 & 29 & 25,400 & 81,900 & 2,180 & 203,000 \\
\hline 1909 & 3,500 & 78,800 & 3,720 & -- & 20,900 & 83 & 31,000 & 109,000 & 2,260 & 249,000 \\
\hline 1910 & 3,380 & 78,800 & 3,680 & -- & 23,000 & 123 & 34,900 & 125,000 & 2,370 & 271,000 \\
\hline 1911 & 3,710 & 75,900 & 3,650 & -- & 20,400 & 133 & 35,500 & 122,000 & 2,590 & 264,000 \\
\hline 1912 & 4,090 & 77,700 & 3,610 & -- & 26,200 & 184 & 38,300 & 124,000 & 2,930 & 277,000 \\
\hline 1913 & 3,810 & 76,200 & 3,580 & -- & 26,400 & 180 & 39,300 & 141,000 & 3,310 & 294,000 \\
\hline 1914 & 3,890 & 72,400 & 4,890 & -- & 18,700 & 152 & 37,300 & 130,000 & 3,560 & 271,000 \\
\hline 1915 & 4,000 & 69,300 & 4,840 & -- & 23,300 & 219 & 38,800 & 131,000 & 4,230 & 276,000 \\
\hline 1916 & 4,140 & 73,000 & 4,800 & -- & 29,800 & 295 & 43,800 & 132,000 & 4,870 & 293,000 \\
\hline 1917 & 4,120 & 69,200 & 5,490 & -- & 30,500 & 297 & 45,400 & 114,000 & 5,360 & 274,000 \\
\hline 1918 & 4,050 & 65,200 & 5,690 & -- & 29,200 & 277 & 40,600 & 93,300 & 5,260 & 244,000 \\
\hline 1919 & 3,860 & 67,400 & 5,670 & -- & 23,300 & 290 & 38,900 & 97,600 & 5,530 & 243,000 \\
\hline 1920 & 3,750 & 67,200 & 6,900 & -- & 30,400 & 307 & 48,200 & 115,000 & 5,790 & 277,000 \\
\hline 1921 & 3,520 & 58,200 & 5,470 & -- & 14,100 & 243 & 36,500 & 104,000 & 5,160 & 228,000 \\
\hline 1922 & 4,180 & 64,100 & 7,130 & -- & 26,800 & 382 & 47,800 & 125,000 & 6,380 & 282,000 \\
\hline 1923 & 4,220 & 70,100 & 8,340 & -- & 34,100 & 486 & 57,900 & 173,000 & 8,390 & 357,000 \\
\hline 1924 & 4,260 & 67,200 & 8,420 & -- & 29,000 & 495 & 59,500 & 194,000 & 10,000 & 373,000 \\
\hline 1925 & 4,560 & 66,300 & 9,450 & -- & 34,600 & 558 & 65,900 & 214,000 & 10,400 & 406,000 \\
\hline 1926 & 4,600 & 65,100 & 10,500 & -- & 36,600 & 653 & 67,100 & 232,000 & 10,600 & 427,000 \\
\hline 1927 & 4,860 & 61,300 & 10,800 & -- & 33,800 & 653 & 66,000 & 255,000 & 11,200 & 444,000 \\
\hline 1928 & 4,750 & 59,500 & 11,300 & -- & 38,400 & 715 & 68,200 & 249,000 & 12,900 & 445,000 \\
\hline 1929 & 4,940 & 61,700 & 12,200 & -- & 41,900 & 750 & 69,600 & 282,000 & 14,400 & 487,000 \\
\hline 1930 & 4,410 & 49,500 & 11,200 & -- & 30,600 & 619 & 60,000 & 255,000 & 13,900 & 425,000 \\
\hline 1931 & 4,280 & 36,200 & 10,300 & -- & 20,300 & 506 & 46,800 & 193,000 & 12,300 & 324,000 \\
\hline 1932 & 3,640 & 27,500 & 8,830 & -- & 11,300 & 387 & 32,000 & 149,000 & 10,600 & 243,000 \\
\hline 1933 & 4,180 & 31,200 & 9,900 & -- & 17,600 & 506 & 33,100 & 143,000 & 11,100 & 250,000 \\
\hline 1934 & 3,620 & 32,900 & 10,200 & -- & 19,400 & 540 & 37,100 & 168,000 & 12,500 & 284,000 \\
\hline 1935 & 4,070 & 38,000 & 11,600 & -- & 25,100 & 657 & 42,100 & 165,000 & 13,200 & 300,000 \\
\hline 1936 & 4,360 & 44,500 & 13,300 & -- & 35,600 & 682 & 54,500 & 249,000 & 15,900 & 418,000 \\
\hline 1937 & 5,010 & 46,800 & 14,500 & -- & 39,300 & 739 & 59,800 & 259,000 & 17,400 & 442,000 \\
\hline 1938 & 4,040 & 41,300 & 12,300 & -- & 25,000 & 520 & 48,300 & 250,000 & 17,200 & 398,000 \\
\hline 1939 & 5,280 & 46,000 & 14,500 & -- & 39,300 & 573 & 58,600 & 304,000 & 18,900 & 487,000 \\
\hline 1940 & 6,420 & 48,700 & 15,200 & -- & 44,900 & 660 & 65,000 & 319,000 & 19,200 & 519,000 \\
\hline 1941 & 7,280 & 56,300 & 18,500 & -- & 61,200 & 922 & 84,100 & 380,000 & 22,400 & 631,000 \\
\hline 1942 & 6,410 & 56,300 & 17,900 & -- & 59,300 & 936 & 89,500 & 402,000 & 22,300 & 655,000 \\
\hline 1943 & 6,640 & 52,100 & 17,600 & -- & 59,100 & 1,040 & 95,900 & 325,000 & 21,700 & 579,000 \\
\hline
\end{tabular}


Table 1. U.S. raw materials put into use annually from 1900 through 2010, by category. Materials embedded in imported goods are not included.-Continued

[In thousand metric tons. Data are rounded to three significant digits. --, negligible or no data]

\begin{tabular}{|c|c|c|c|c|c|c|c|c|c|c|}
\hline \multirow[b]{2}{*}{ Year } & \multirow[b]{2}{*}{ Agriculture } & \multicolumn{3}{|c|}{ Forestry } & \multicolumn{4}{|c|}{ Metals and minerals } & \multirow{2}{*}{$\begin{array}{c}\text { Nonrenewable } \\
\text { organics }\end{array}$} & \multirow{2}{*}{$\begin{array}{c}\text { Total of } \\
\text { materials }\end{array}$} \\
\hline & & Wood & $\begin{array}{l}\text { Paper and } \\
\text { paperboard }\end{array}$ & $\begin{array}{l}\text { Recycled } \\
\text { paper }\end{array}$ & $\begin{array}{l}\text { Primary } \\
\text { metals }\end{array}$ & $\begin{array}{l}\text { Recycled } \\
\text { metals }\end{array}$ & $\begin{array}{l}\text { Industrial } \\
\text { minerals }\end{array}$ & $\begin{array}{l}\text { Construction } \\
\text { materials }\end{array}$ & & \\
\hline 1944 & 6,260 & 50,400 & 17,600 & -- & 59,000 & 1,090 & 88,800 & 281,000 & 23,100 & 527,000 \\
\hline 1945 & 6,070 & 44,500 & 17,800 & -- & 56,300 & 1,090 & 90,100 & 279,000 & 25,600 & 521,000 \\
\hline 1946 & 6,800 & 51,400 & 20,400 & -- & 48,700 & 1,020 & 107,000 & 353,000 & 27,500 & 616,000 \\
\hline 1947 & 6,800 & 53,000 & 22,400 & -- & 60,500 & 1,280 & 131,000 & 394,000 & 28,900 & 698,000 \\
\hline 1948 & 7,310 & 54,500 & 23,700 & -- & 68,600 & 30,700 & 140,000 & 437,000 & 29,500 & 791,000 \\
\hline 1949 & 6,630 & 47,700 & 22,400 & -- & 60,400 & 23,800 & 132,000 & 438,000 & 28,300 & 759,000 \\
\hline 1950 & 7,100 & 56,200 & 26,300 & -- & 76,800 & 32,600 & 151,000 & 501,000 & 31,500 & 883,000 \\
\hline 1951 & 6,860 & 53,600 & 27,700 & -- & 84,500 & 31,700 & 166,000 & 555,000 & 34,600 & 959,000 \\
\hline 1952 & 6,720 & 53,800 & 26,300 & -- & 70,400 & 32,600 & 163,000 & 600,000 & 33,100 & 986,000 \\
\hline 1953 & 6,820 & 53,500 & 28,400 & -- & 84,800 & 31,700 & 171,000 & 605,000 & 34,700 & $1,020,000$ \\
\hline 1954 & 6,680 & 52,900 & 28,500 & -- & 65,600 & 24,600 & 173,000 & 813,000 & 35,300 & $1,200,000$ \\
\hline 1955 & 6,880 & 54,700 & 31,500 & -- & 88,000 & 33,700 & 194,000 & 881,000 & 38,700 & $1,330,000$ \\
\hline 1956 & 6,840 & 55,100 & 33,100 & -- & 86,400 & 38,600 & 202,000 & 942,000 & 41,500 & $1,410,000$ \\
\hline 1957 & 5,990 & 48,700 & 32,000 & -- & 81,600 & 29,500 & 191,000 & 976,000 & 40,900 & $1,410,000$ \\
\hline 1958 & 5,800 & 49,800 & 31,900 & -- & 63,500 & 22,200 & 185,000 & $1,030,000$ & 42,700 & $1,430,000$ \\
\hline 1959 & 6,390 & 54,600 & 35,100 & -- & 77,600 & 27,600 & 212,000 & $1,100,000$ & 46,300 & $1,560,000$ \\
\hline 1960 & 6,380 & 49,100 & 27,200 & 8,400 & 76,500 & 24,900 & 207,000 & $1,120,000$ & 47,300 & $1,560,000$ \\
\hline 1961 & 6,740 & 48,500 & 28,200 & 8,400 & 72,600 & 24,100 & 206,000 & $1,100,000$ & 48,600 & $1,540,000$ \\
\hline 1962 & 7,220 & 50,600 & 30,000 & 8,460 & 78,500 & 24,400 & 218,000 & $1,140,000$ & 51,800 & $1,610,000$ \\
\hline 1963 & 7,400 & 54,200 & 30,700 & 8,970 & 84,800 & 28,300 & 231,000 & $1,270,000$ & 52,800 & $1,770,000$ \\
\hline 1964 & 7,650 & 57,000 & 33,000 & 9,190 & 94,200 & 30,600 & 245,000 & $1,340,000$ & 55,500 & $1,870,000$ \\
\hline 1965 & 7,120 & 60,700 & 31,700 & 9,560 & 108,000 & 34,400 & 259,000 & $1,420,000$ & 59,400 & $1,990,000$ \\
\hline 1966 & 7,540 & 61,000 & 34,900 & 9,880 & 107,000 & 35,300 & 273,000 & $1,480,000$ & 64,000 & $2,070,000$ \\
\hline 1967 & 8,020 & 58,600 & 34,900 & 9,280 & 101,000 & 38,300 & 271,000 & $1,430,000$ & 64,500 & $2,010,000$ \\
\hline 1968 & 9,100 & 60,900 & 37,200 & 9,600 & 114,000 & 38,300 & 282,000 & $1,460,000$ & 70,600 & $2,080,000$ \\
\hline 1969 & 6,930 & 61,000 & 38,200 & 11,200 & 101,000 & 42,000 & 291,000 & $1,520,000$ & 75,700 & $2,140,000$ \\
\hline 1970 & 6,560 & 60,200 & 36,800 & 11,400 & 96,000 & 38,100 & 287,000 & $1,530,000$ & 80,400 & $2,150,000$ \\
\hline 1971 & 6,750 & 64,700 & 37,300 & 11,700 & 101,000 & 38,900 & 290,000 & $1,510,000$ & 81,800 & $2,150,000$ \\
\hline 1972 & 7,540 & 66,900 & 40,500 & 12,400 & 106,000 & 46,600 & 309,000 & $1,540,000$ & 85,400 & $2,220,000$ \\
\hline 1973 & 5,410 & 66,700 & 42,100 & 13,800 & 122,000 & 53,000 & 322,000 & $1,730,000$ & 92,600 & $2,440,000$ \\
\hline 1974 & 4,770 & 57,900 & 40,900 & 14,200 & 118,000 & 57,100 & 316,000 & $1,650,000$ & 91,500 & $2,350,000$ \\
\hline 1975 & 5,840 & 53,500 & 34,900 & 11,900 & 84,900 & 44,100 & 277,000 & $1,420,000$ & 79,900 & $2,020,000$ \\
\hline 1976 & 5,880 & 60,300 & 39,700 & 14,000 & 102,000 & 47,200 & 297,000 & $1,500,000$ & 89,200 & $2,160,000$ \\
\hline 1977 & 5,610 & 66,100 & 40,800 & 14,800 & 104,000 & 46,600 & 314,000 & $1,590,000$ & 97,000 & $2,280,000$ \\
\hline 1978 & 5,340 & 68,800 & 43,100 & 15,200 & 116,000 & 48,300 & 332,000 & $1,730,000$ & 99,100 & $2,450,000$ \\
\hline 1979 & 5,790 & 67,500 & 44,200 & 16,300 & 85,400 & 55,300 & 340,000 & $1,750,000$ & 106,000 & $2,470,000$ \\
\hline 1980 & 5,040 & 58,800 & 42,500 & 16,300 & 94,600 & 49,800 & 312,000 & $1,500,000$ & 113,000 & $2,190,000$ \\
\hline 1981 & 4,940 & 54,700 & 44,000 & 16,100 & 103,000 & 46,600 & 295,000 & $1,330,000$ & 101,000 & $2,000,000$ \\
\hline 1982 & 5,330 & 55,300 & 41,800 & 15,500 & 85,400 & 34,100 & 258,000 & $1,180,000$ & 91,300 & $1,770,000$ \\
\hline 1983 & 5,260 & 66,400 & 46,100 & 17,000 & 83,200 & 40,400 & 275,000 & $1,300,000$ & 90,800 & $1,920,000$ \\
\hline 1984 & 5,650 & 71,500 & 49,500 & 18,600 & 97,200 & 44,000 & 311,000 & $1,480,000$ & 95,500 & $2,170,000$ \\
\hline 1985 & 6,550 & 72,900 & 48,800 & 18,500 & 94,900 & 47,100 & 310,000 & $1,540,000$ & 97,000 & $2,240,000$ \\
\hline 1986 & 6,170 & 77,900 & 50,200 & 20,400 & 89,200 & 47,900 & 301,000 & $1,630,000$ & 97,300 & $2,320,000$ \\
\hline 1987 & 6,480 & 82,700 & 52,700 & 21,800 & 93,500 & 53,000 & 309,000 & $1,800,000$ & 107,000 & $2,530,000$ \\
\hline
\end{tabular}


Table 1. U.S. raw materials put into use annually from 1900 through 2010, by category. Materials embedded in imported goods are not included.-Continued

[In thousand metric tons. Data are rounded to three significant digits. --, negligible or no data]

\begin{tabular}{|c|c|c|c|c|c|c|c|c|c|c|}
\hline \multirow[b]{2}{*}{ Year } & \multirow[b]{2}{*}{ Agriculture } & \multicolumn{3}{|c|}{ Forestry } & \multicolumn{4}{|c|}{ Metals and minerals } & \multirow{2}{*}{$\begin{array}{c}\text { Nonrenewable } \\
\text { organics }\end{array}$} & \multirow{2}{*}{$\begin{array}{c}\text { Total of } \\
\text { materials }\end{array}$} \\
\hline & & Wood & $\begin{array}{c}\text { Paper and } \\
\text { paperboard }\end{array}$ & $\begin{array}{c}\text { Recycled } \\
\text { paper }\end{array}$ & $\begin{array}{l}\text { Primary } \\
\text { metals }\end{array}$ & $\begin{array}{l}\text { Recycled } \\
\text { metals }\end{array}$ & $\begin{array}{l}\text { Industrial } \\
\text { minerals }\end{array}$ & $\begin{array}{c}\text { Construction } \\
\text { materials }\end{array}$ & & \\
\hline 1988 & 5,880 & 80,300 & 52,900 & 23,700 & 78,400 & 57,300 & 323,000 & $1,870,000$ & 110,000 & $2,600,000$ \\
\hline 1989 & 5,690 & 81,000 & 51,700 & 24,600 & 93,700 & 55,700 & 319,000 & $1,810,000$ & 108,000 & $2,550,000$ \\
\hline 1990 & 5,480 & 77,900 & 51,300 & 26,400 & 94,300 & 58,900 & 320,000 & $1,840,000$ & 115,000 & $2,590,000$ \\
\hline 1991 & 5,460 & 71,000 & 48,000 & 28,300 & 85,200 & 52,800 & 301,000 & $1,620,000$ & 116,000 & $2,330,000$ \\
\hline 1992 & 5,240 & 74,800 & 48,600 & 30,800 & 91,900 & 54,600 & 310,000 & $1,790,000$ & 121,000 & $2,530,000$ \\
\hline 1993 & 7,110 & 76,200 & 50,400 & 32,200 & 79,400 & 58,700 & 316,000 & $1,890,000$ & 124,000 & $2,640,000$ \\
\hline 1994 & 7,240 & 77,400 & 50,300 & 36,000 & 106,000 & 62,900 & 339,000 & $2,020,000$ & 130,000 & $2,830,000$ \\
\hline 1995 & 5,650 & 77,100 & 48,900 & 38,300 & 112,000 & 64,000 & 343,000 & $2,070,000$ & 131,000 & $2,890,000$ \\
\hline 1996 & 5,740 & 77,800 & 46,400 & 39,100 & 118,000 & 62,200 & 356,000 & $2,150,000$ & 133,000 & $2,990,000$ \\
\hline 1997 & 6,050 & 78,800 & 50,100 & 39,900 & 123,000 & 64,500 & 361,000 & $2,270,000$ & 138,000 & $3,130,000$ \\
\hline 1998 & 5,680 & 80,600 & 50,700 & 40,900 & 128,000 & 61,400 & 370,000 & $2,480,000$ & 145,000 & $3,360,000$ \\
\hline 1999 & 5,770 & 83,100 & 52,700 & 42,500 & 127,000 & 58,600 & 384,000 & $2,530,000$ & 151,000 & $3,440,000$ \\
\hline 2000 & 5,280 & 82,300 & 50,600 & 42,900 & 130,000 & 61,400 & 373,000 & $2,560,000$ & 141,000 & $3,450,000$ \\
\hline 2001 & 4,740 & 80,100 & 45,600 & 42,600 & 115,000 & 59,800 & 365,000 & $2,620,000$ & 133,000 & $3,460,000$ \\
\hline 2002 & 4,920 & 82,600 & 45,000 & 43,200 & 109,000 & 60,700 & 357,000 & $2,540,000$ & 135,000 & $3,380,000$ \\
\hline 2003 & 4,440 & 81,600 & 46,300 & 44,700 & 115,000 & 60,600 & 371,000 & $2,590,000$ & 132,000 & $3,440,000$ \\
\hline 2004 & 4,530 & 89,100 & 48,900 & 45,600 & 126,000 & 63,800 & 392,000 & $2,760,000$ & 142,000 & $3,670,000$ \\
\hline 2005 & 4,180 & 91,300 & 45,900 & 46,500 & 122,000 & 62,800 & 403,000 & $2,870,000$ & 137,000 & $3,780,000$ \\
\hline 2006 & 3,880 & 85,700 & 44,600 & 48,400 & 127,000 & 64,200 & 384,000 & $3,000,000$ & 135,000 & $3,890,000$ \\
\hline 2007 & 3,850 & 76,700 & 41,300 & 49,300 & 122,000 & 69,900 & 370,000 & $2,800,000$ & 134,000 & $3,670,000$ \\
\hline 2008 & 3,050 & 57,600 & 37,900 & 47,000 & 107,000 & 78,400 & 346,000 & $2,450,000$ & 123,000 & $3,250,000$ \\
\hline 2009 & 3,810 & 48,600 & 28,800 & 45,400 & 67,300 & 74,400 & 277,000 & $1,950,000$ & 115,000 & $2,610,000$ \\
\hline 2010 & 3,620 & 49,100 & 30,600 & 46,800 & 85,400 & 70,500 & 294,000 & $1,870,000$ & 116,000 & $2,570,000$ \\
\hline
\end{tabular}




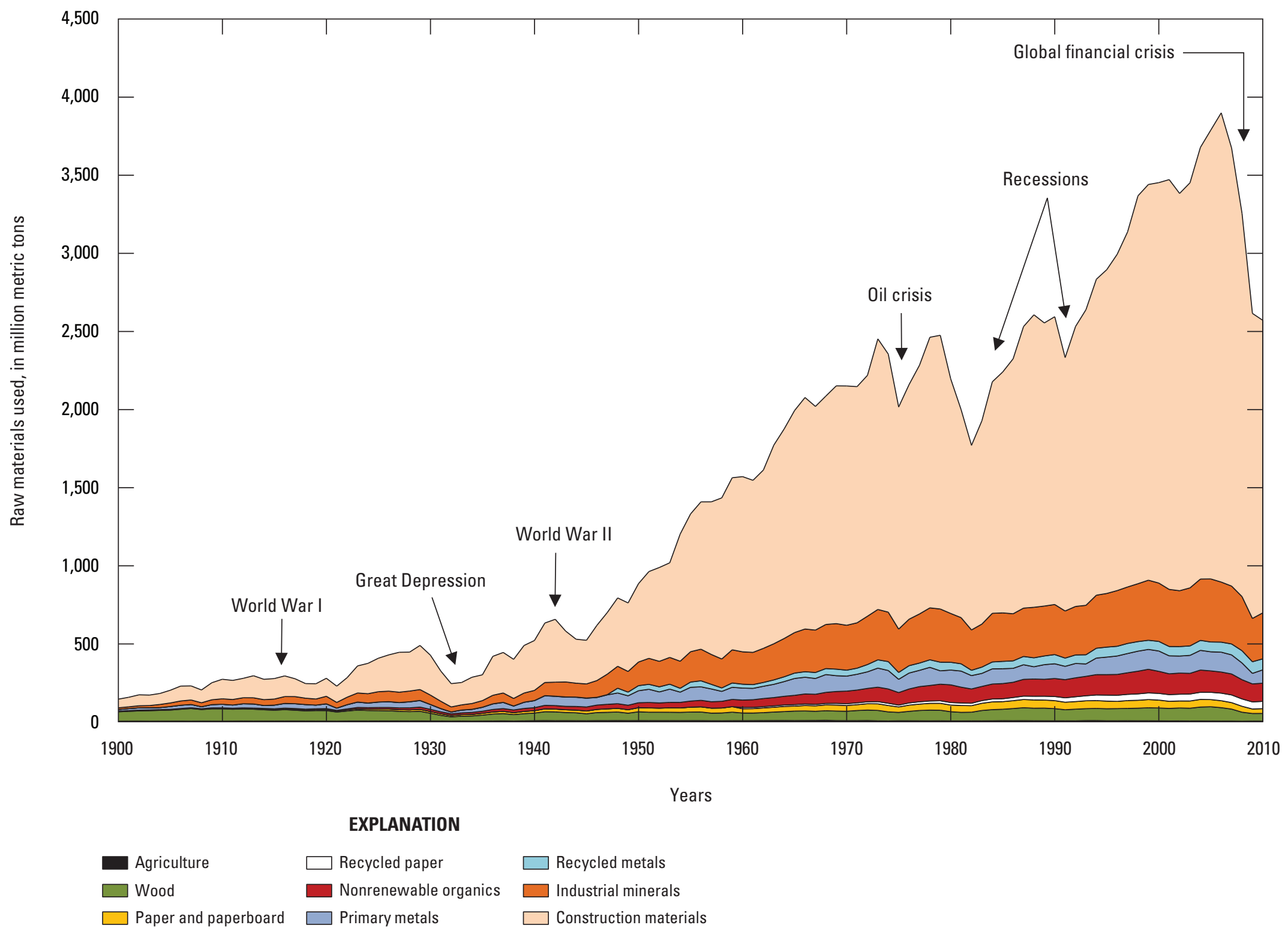

Figure 2. U.S. raw materials put into use annually from 1900 through 2010. Materials embedded in imported goods are not included. [In million metric tons] 


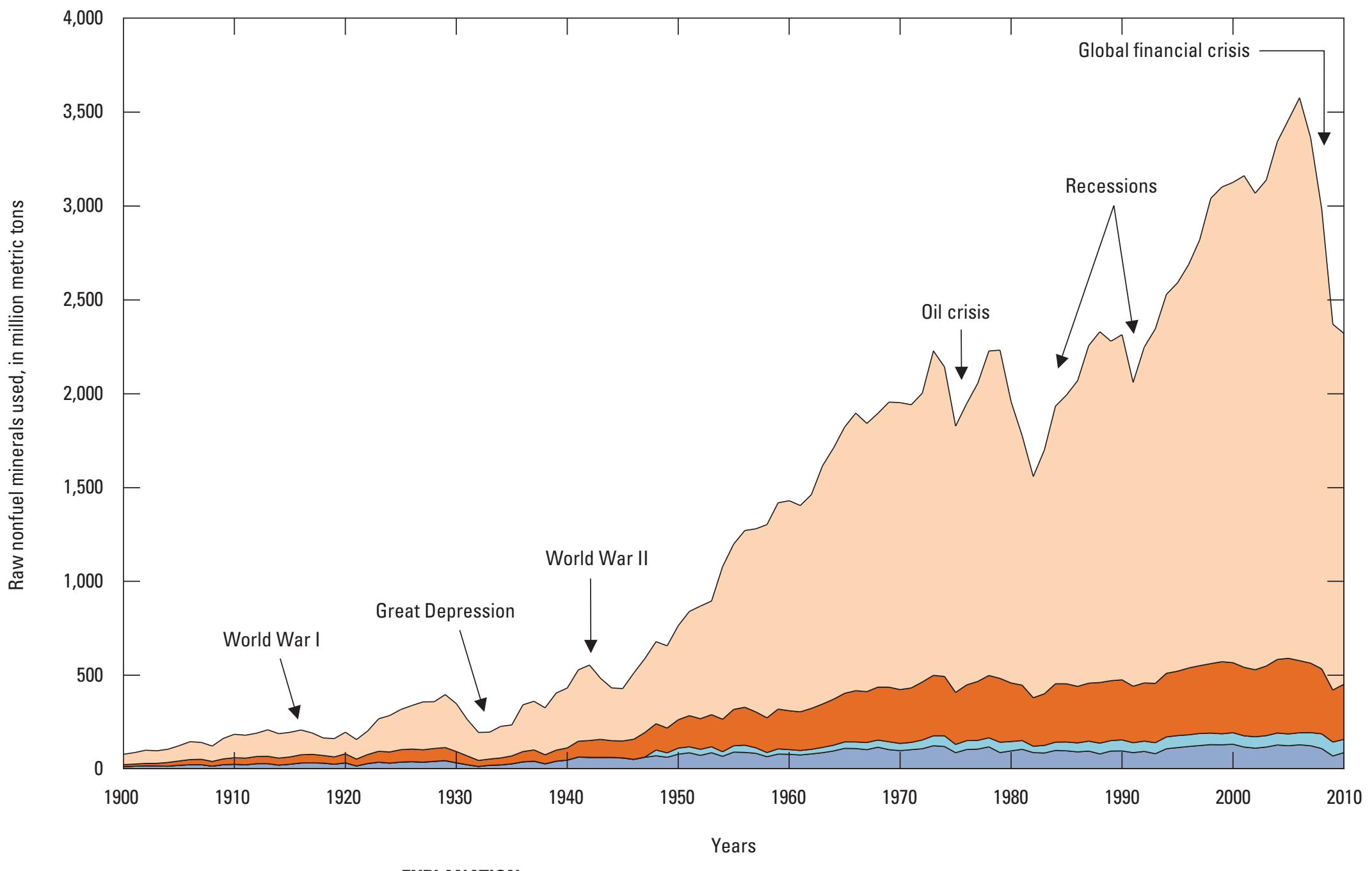

\section{EXPLANATION}

$\square$ Primary metals $\square$ Recycled metals $\square$ Industrial minerals $\square$ Construction materials

Figure 3. U.S. raw nonfuel minerals put into use annually from 1900 through 2010. Mineral materials embedded in imported goods are not included. [In million metric tons] 